\section{Studien richtig planen und aufsetzen}

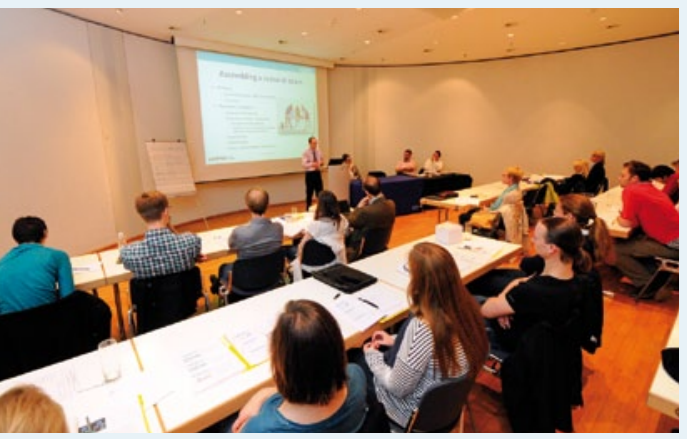

Unterstützt von der neuen Fortbildungsinitiative „GABA Oral Health Network“ hat die ebenfalls neu ins Leben gerufene DGPZM (Deutsche Gesellschaft für Präventivzahnmedizin) zu einer Weiterbildung nach Frankfurt am Main eingeladen. 26 wissenschaftliche Mitarbeiter und Forscher von Universitäten nutzten die Gelegenheit und informierten sich im Museum für Kommunikation über „Clinical Studies: From the idea to first patient first visit“. Die Teilnehmer von deutschen, schweizerischen und österreichischen Universitäten erhielten auf dem Workshop Informationen rund um die Planung, das Aufsetzen und die Veröffentlichung wissenschaftlicher Studien. Prof. Carolina Ganß, Präsidentin der DGPZM, führte durch die Veranstaltung. Eric
Die Teilnehmer folgten dem Vortrag von Prof. Needleman (Eastman Dental Institute, London).

Huber (Tropeninstitut Basel) sprach über rechtliche Rahmenbedingungen und praktische Aspekte in der Studienvorbereitung, Prof. Ian Needleman (UCL; Eastman Dental Institute, London) gab einen Überblick über Qualitätskriterien und Entwicklung von Studienplänen bis zur korrekten Zusammenfassung wissenschaftlicher Studien, und PD Dr. Christian Heumann (LMU, Abteilung für Statistik, München) erläuterte die erforderlichen statistischen Ansätze in der Studienplanung. Mit einem Abendessen klang die Veranstaltung aus.

Das „Oral Health Network“ ist eine Fortbildungsinitiative von GABA/Colgate, unter deren Dach regelmäßig Fortbildungen und Expertengespräche stattfinden. „Mit dieser Initiative möchten wir neue Lösungsansätze für aktuelle zahnmedizinische Fragestellungen diskutieren, Experten miteinander vernetzen und den wissenschaftlichen Nachwuchs fördern“, fasst Dr. Barbara Egger von GABA die Zielsetzung der Initiative zusammen.

Dieser Beitrag ist entstanden mit freundlicher Unterstützung der

GABA GmbH, Lörrach

\title{
Roadshow
}

\section{Erfolgreicher Auftakt}

Erfolgreicher Auftakt in Dresden: Unter dem Titel „Parodontaltherapie - ein Erfolg ohne Nebenwirkungen?!" startete die jüngste GABA-Roadshow mit hochkarätigen Referenten. Unter Leitung von Prof. Thomas Hoffmann (Dresden) erfuhren die Zuhörer Wissenswertes über die gingivale Rezession als eine alltägliche multidisziplinäre Herausforderung. Oberärztin Dr. Katrin Nickels (Frankfurt) gab Hinweise zu der Frage, wie bei Paropatienten Schmerzfreiheit und Zahnerhalt möglich sind. Zu guter Letzt verriet Dentalhygienikerin Susanne Graack (Hamburg) Tipps und Tricks für eine erfolgreiche Prophylaxesitzung. Mit einer angeregten Diskussion und einem Imbiss endete die Veranstaltung im Internationalen Congress Center in Dresden. Im Fokus dieser Fortbildung steht das vielschichtige Thema der gingivalen Re- zession. Praktische Erkenntnisse und Tipps aus dem Arbeitsalltag runden das Informationsangebot ab.

Hier die weiteren Termine: 7.9., Hamburg; 12.9., Düsseldorf; 26.9., Stuttgart; 19.10., Berlin; 21.11., Hannover; 29.11., Basel.

Beginn ist in Deutschland jeweils um 17, in Basel um 16 Uhr. Jede Veranstaltung dauert etwa 3h, die Teilnahme kostet für Zahnärzte $105 €$ in Deutschland bzw. 136CHF in der Schweiz, für Praxismitarbeiter $82 €$ in Deutschland bzw. 107CHF in der Schweiz. Weitere Details sowie Anmeldung unter www.gaba-dent.de/fortbildung oder Tel. 03641/3116160. Unter der angegebenen URL sind ab sofort auch Bilder der Auftaktveranstaltung zu sehen.

Nach einer Pressemitteilung der

GABA GmbH, Lörrach

\section{Zahnerosionen}

\section{noch stärker ins}

Bewusstsein rücken

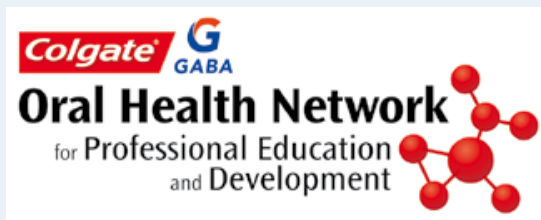

Zum 3. und letzten Mal luden Prof. Adrian Lussi und GABA internationale Wissenschaftler zu einem Erosions-Workshop nach Bern ein. 14 Teilnehmer folgten der Einladung und erlebten 3 spannende, informationsreiche Tage im Herzen der Schweiz. Auf dem Programm stand der aktuelle Wissens- und Forschungsstand zur Erosion. Im Labor und am Patientenstuhl wurden sowohl Laboruntersuchungsmethoden als auch das Vorgehen bei Diagnose und Therapie am Patienten vermittelt.

Sowohl die Forschungslage als auch die Behandlungssituation zur Erosion in Europa sei immer noch nicht etabliert, befanden die Teilnehmer einmütig. Nach wie vor bedürfe es weiterer Bemühungen seitens der Wissenschaft und der Industrie, um die Indikation Erosion noch stärker in das Bewusstsein der Zahnarztpraxen zu rücken. „Außerdem bestehen nach wie vor Schwierigkeiten bei der Diagnostik, obwohl es mit dem BEWE (Basic Erosive Wear Examination) einen etablierten und pragmatischen Standard gibt“, ergänzt Dr. Barbara Egger von GABA. „Auch das Wissen um Risikofaktoren und Behandlungsmöglichkeiten ist noch nicht weit genug verbreitet." Um hier einen Beitrag zu leisten, hat GABA seit 2010 insgesamt 3 Erosions-Workshops mit Prof. Lussi organisiert, in denen theoretische und praktische Kenntnisse vermittelt wurden.

Die Veranstaltung in Bern war ein weiterer Baustein der von GABA/Colgate ins Leben gerufenen Fortbildungsinitiative „Oral Health Network“. In ihrem Rahmen finden regelmäßig Fortbildungen und Expertengespräche statt mit dem Ziel, neue Lösungsansätze für aktuelle zahnmedizinische Fragestellungen zu diskutieren und etablierte wie auch angehende Experten miteinander zu vernetzen.

Nach einer Pressemitteilung der

GABA GmbH, Lörrach

Internet: www.gaba.com 